\title{
Microwave absorption on a thin film
}

\author{
Herman Bosman, Y. Y. Lau, a) and R. M. Gilgenbach \\ Department of Nuclear Engineering and Radiological Sciences, University of Michigan, Ann Arbor, \\ Michigan 48109-2104
}

(Received 11 September 2002; accepted 6 January 2003)

\begin{abstract}
With the use of a simple model, it is shown that a thin film of contaminant on a microwave window may absorb up to $50 \%$ of the incident power, even if the film thickness is only a small fraction of its resistive skin depth. This unexpectedly large amount of absorption is conjectured to have played a significant role in window failure. The temperature rise in a thin film is estimated. (C) 2003 American Institute of Physics. [DOI: 10.1063/1.1556969]
\end{abstract}

There are several mechanisms that may lead to the failure of a high power microwave window. ${ }^{1}$ The most troublesome type of window failure occurs unexpectedly, at a power level considered "safe" in the design. Such failures are usually accompanied by some strong local heating on the surface. Multipactor may cause local heating. ${ }^{2-4}$ The multipactoring electrons may also cause outgassing, subsequent ionization, and flashover on the dielectric window. ${ }^{1,5}$ Measures to reduce multipactor are well-known, for example, coating a slightly conducting thin film may prevent dielectric charging which is the physical origin of multipactor. ${ }^{6,7}$ Simple estimates show that multipactor is unlikely to be a threat to microwave windows at frequencies higher than about $30 \mathrm{GHz}{ }^{1}$

For high power millimeter wave sources, ${ }^{8,9}$ such as the megawatt, long pulse (10 s), $170 \mathrm{GHz}$ gyrotron, the expensive diamond windows do fail occasionally. While multipactor is unlikely to be the reason for these high frequency window failures, neither is the graphitization of diamond, as we have recently concluded that the temperature increase, as a result of the heating of the graphite impurities, is insufficient to convert more diamond into graphite. ${ }^{10}$ These negative results motivated the present investigation of the heating of a thin film of contaminants on a window surface.

For simplicity, consider the absorption of a uniform thin film of contaminants on the window. The film, of thickness $L$, faces the incident wave [Fig. 1(a)]. We assume that the incident wave is a plane wave of frequency $\omega$, and that the window itself is lossless and is perfectly matched. That is, in the absence of the thin film, an incident wave undergoes $100 \%$ transmission, with zero reflection and zero absorption from the window. Thus, to the right side of the thin film, the wave essentially sees a vacuum [Fig. 1(b)].

Let $\varepsilon=\varepsilon(\omega)$ be the real part of the dielectric function and $\sigma=\sigma(\omega)$ be the electrical conductivity of the thin film. This thin film is assumed to be nonmagnetic, i.e., $\mu=\mu_{0}$, the free space permeability. The fraction of the incident power, $A$, that is absorbed within this lossy thin film may easily be calculated from $A=1-|R|^{2}-|T|^{2}$, where $R$ is the reflection coefficient and $T$ is the transmission coefficient, in complex amplitudes. For the geometry shown in Fig. 1(b), $R$ and $T$ may be obtained from standard textbooks: ${ }^{11} R=\left(Z_{R}\right.$

${ }^{a)}$ Electronic mail: yylau@umich.edu
$-1) /\left(Z_{R}+1\right), \quad T=(1+R) \cos (k L)-j\left(Z / Z_{0}\right)(1-R) \sin (k L)$, where $j$ is the unit imaginary number, $Z_{R}=[1$ $\left.+j\left(Z / Z_{0}\right) \tan (k L)\right] /\left[1+j\left(Z_{0} / Z\right) \tan (k L)\right], \quad Z_{0}=\left(\mu_{0} / \varepsilon_{0}\right)^{1 / 2}$ $=377 \Omega$ is the free space impedance, $Z=\left[\mu_{0} /(\varepsilon\right.$ $+\sigma / j \omega)]^{1 / 2}$ is the characteristic impedance of the thin film, and $k=\omega\left[\mu_{0}(\varepsilon+\sigma / j \omega)\right]^{1 / 2}$ is its complex wave number.

Figure 2 shows the absorption coefficient, $A$, as a function of the film thickness, $L$. In Fig. 2, the film thickness is normalized to the scale length

$$
s=\frac{2}{\sigma Z_{0}},
$$

which depends only on the electrical conductivity, $\sigma$; and the skin depth $\delta=\left(2 / \omega \mu_{0} \sigma\right)^{1 / 2}$ is normalized to the free space wavelength $\lambda=2 \pi c / \omega$, with $c$ being the speed of light in vacuum. Note that $\delta / \lambda=s / 2 \pi \delta$, and that $L / \delta=2 \pi(L / s)$ $\times(\delta / \lambda)$. It is seen from Fig. 2 that the thin film may absorb up to $50 \%$ of the incident power even if the thin film thickness $L$ is much less than the skin depth $\delta$. This result is counter-intuitive because, if $L \ll \delta$, the thin film might be
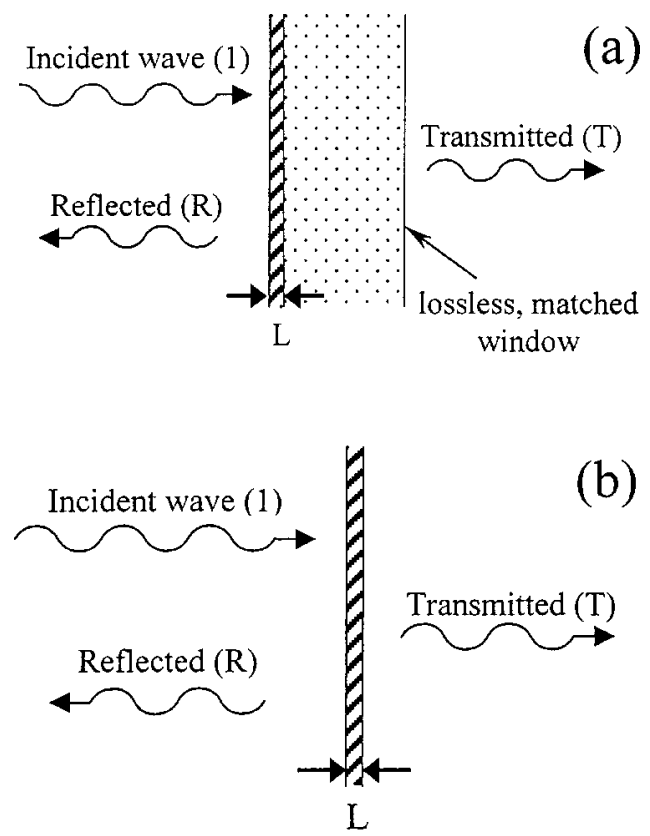

FIG. 1. (a) A thin film in front a lossless, matched window. (b) An equivalent model. 


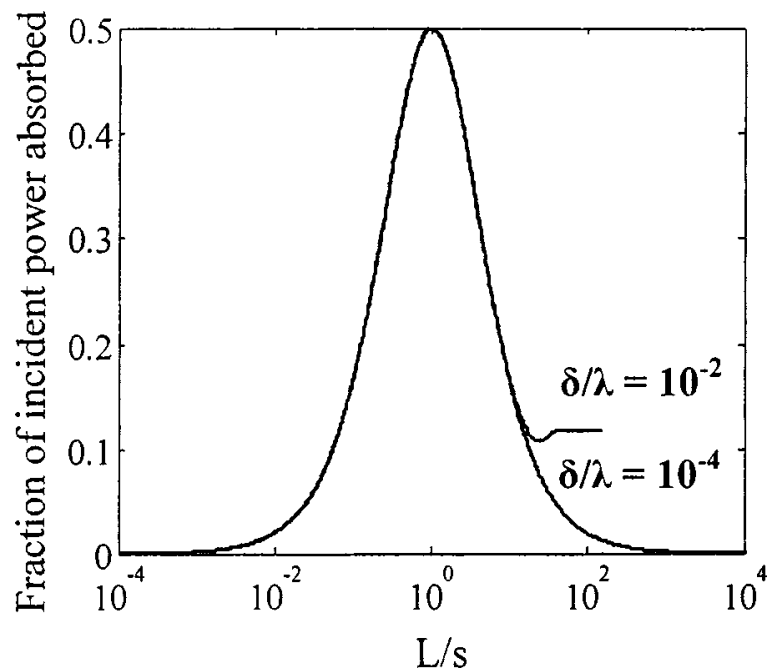

FIG. 2. The fraction of incident power absorbed by the thin film, under the assumption $\omega \varepsilon \ll \sigma$.

expected to appear "transparent" to the incident wave, leading to little absorption. This feature is present in the space cloth/Salisbury sheet, ${ }^{12}$ however.

To understand the unexpectedly large amount of absorption by the thin film, let us focus at the regime, $L \ll \delta \ll \lambda$. The first inequality may be considered as the definition of a thin film. The last inequality is easily satisfied whenever $\omega \varepsilon \ll \sigma$, which is satisfied if $\sigma>10^{3} / \Omega \mathrm{m}, \varepsilon / \varepsilon_{0}$ is of order 10 or less, and $\omega / 2 \pi$ is less than $200 \mathrm{GHz}$.

For $L \ll \delta$, the tangential electric field is approximately continuous across the thin film [Fig. 1(b)]. Thus, in the transmission line analogue [Fig. 3(a)], the thin film may be modeled by a lumped resistor, $R_{\mathrm{eq}}$. The transmission line voltage, which represents the tangential electric field, is
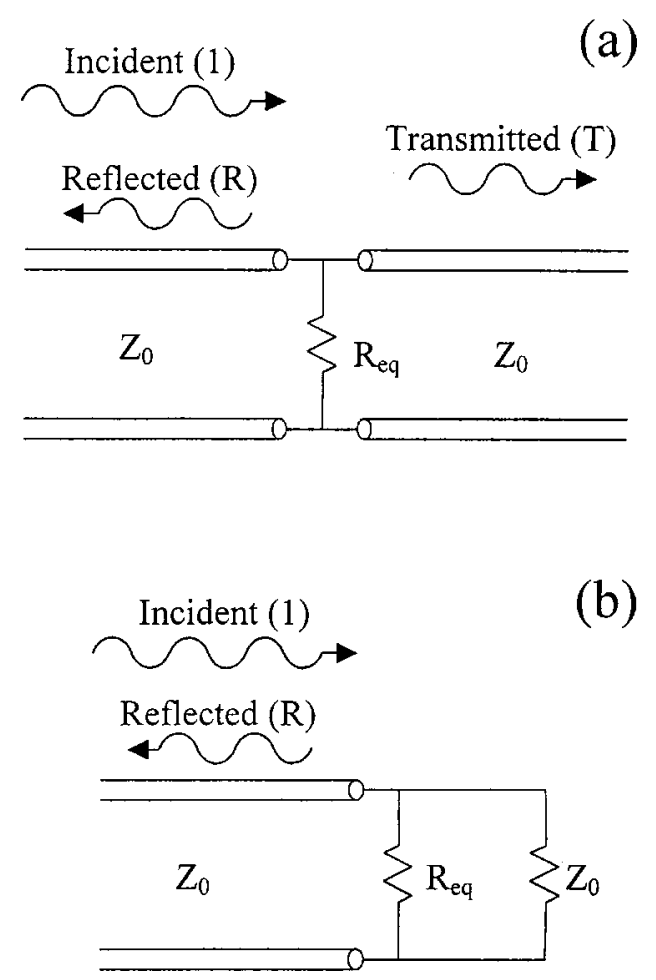

FIG. 3. (a) The equivalent transmission line model for the thin film. (b) Further simplification. continuous on either side of this lumped resistor $R_{\text {eq }}$, which is approximately given by

$$
R_{\text {eq }}=\frac{1}{\sigma L} \text {. }
$$

In Eq. (2), a larger value of $L$ means a larger width for current conduction in the thin film, and hence, a smaller equivalent resistance. It is plain that Fig. 3(b) is equivalent to Fig. 3(a), as far as the incident wave is concerned.

It is now a simple matter to calculate the fraction of the incident rf power $(A)$ that is absorbed by $R_{\text {eq }}$ in Fig. 3(b). We find

$$
A=\frac{2 \xi}{(1+\xi)^{2}},
$$

where

$$
\xi=\frac{Z_{0}}{2 R_{\mathrm{eq}}}=\frac{L}{S} .
$$

In writing the last expression of Eq. (4), we have used Eqs. (1) and (2). It is easy to show from Eq. (3) that the maximum value of $A$ is 0.5 , occuring when $\xi=1$, i.e., when $L=s$ according to Eq. (4), as also shown in Fig. 2. When $A=0.5$, one quarter of the incident power is reflected, and the remaining quarter is transmitted. With Eq. (4), the bell-shaped curve shown in Fig. 2 is easily understood by considering the limits $R_{\text {eq }} \ll Z_{0}$ and $R_{\text {eq }} \gg Z_{0}$ in Fig. 3(b). In these limits, $R_{\text {eq }}$ behaves either like a short or an open circuit, and it cannot absorb significant $\mathrm{rf}$ power in either case.

The simple results given earlier allow us to estimate the temperature rise in a thin, cylindrical disk of contaminants attached to the window surface. We assume that this contaminant disk has a width $L$ and radius $a$, with $L \ll a$. The amount of rf power absorbed in this patch of contaminant is approximately given by $P=A\left(S \pi a^{2}\right)$, where $S$ (in $\mathrm{W} / \mathrm{m}^{2}$ ) is the average Poynting flux of the incident wave, and $A$ is the absorption coefficient given by Eq. (3). In the steady state, the temperature increase, $T$, within this disk is governed by the time-independent diffusion equation

$$
\nabla^{2} T(\mathbf{r})=-\frac{p(\mathbf{r})}{\kappa},
$$

where $\kappa$ is the thermal conductivity (in $\mathrm{W} / \mathrm{m} \mathrm{K}$ ) of the disk, and $p=P /\left(\pi a^{2} L\right)$ is the average power density (in $\mathrm{W} / \mathrm{m}^{3}$ ) deposited onto the disk.

Equation (5) is identical to the Poisson equation for the electrostatic potential $\phi(T)$, due to a disk of charge with charge density $\rho(p)$ in free space $\varepsilon_{0}(\kappa)$. The peak potential $\phi(T)$ occurs at the center of the disk. For a thin disk of charge in free space with $L \ll a$, the maximum potential at the center of the disk is easily shown to be $\rho a L / 2 \varepsilon_{0}$ by summing the contributions from the concentric rings of charge that make up the disk. Replacing $\rho$ by $p$ and $\varepsilon_{0}$ by $\kappa$, we arrive at the following estimate of the maximum temperature increase on the contaminant disk

$$
\begin{aligned}
\Delta T= & 100^{\circ} \mathrm{K} \times \frac{a}{1 \mathrm{~mm}} \times \frac{L / s}{(1+L / s)^{2}} \times \frac{S}{10^{8} \mathrm{~W} / \mathrm{m}^{2}} \\
& \times \frac{1}{\kappa /\left(10^{3} \mathrm{~W} \mathrm{~m}^{-1} \mathrm{~K}^{-1}\right)} .
\end{aligned}
$$


Equation (6) only gives a very rough estimate on the temperature increase, considering the great uncertainties in the thermal, electrical, and geometrical properties of the thin film contaminant, all of which enter Eq. (6). Moreover, Eq. (6) was derived from the particular solution to Eq. (5); the homogeneous solution, which depends on the boundary conditions imposed on the contaminant surface, has been ignored. We have also assumed that the simple model may be applied to a thin film. Despite these limitations, the order of magnitude displayed in Eq. (6) for the temperature increase allows the tentative conclusion that a thin layer of contaminant may lead to an unexpectedly high level of rf absorption and possibly to window failure. Recent experiences on window failure ${ }^{13-15}$ seem to be consistent with these findings and point to the importance of maintaining a clean window as a preventive measure against failure.

The authors wish to thank A. Theiss, T. V. George, R. Temkin, R. True, C. Armstrong, J. W. Luginsland, J. Booske, and $\mathrm{H}$. Jory and for many stimulating discussions. This work was supported by DOE Grant DE-FG02-98ER54475, AFOSR, DUSD ( $\mathrm{S} \& \mathrm{~T}$ ) under the Innovative Microwave Vacuum Electronics MURI Program, managed by the Air Force Office of Scientific Research under Grant F49620-991-0297, and Northrop Grumman Industrial Affiliates Program.
${ }^{1}$ A. A. Neuber, L. Laurent, Y. Y. Lau, and H. Krompholz, in High-Power Microwave Sources and Technologies, edited by R. J. Barker and E. Schamiloglu (IEEE, New York, 2001), Chap. 10, p. 325.

${ }^{2}$ D. H. Priest and R. C. Talcott, IRE Trans. Electron Devices ED-8, 243 (1961).

${ }^{3}$ R. A. Rimmer, Ph.D. dissertation, Lancaster University, Lancaster, UK, 1988.

${ }^{4}$ L. K. Ang, Y. Y. Lau, R. A. Kishek, and R. M. Gilgenbach, IEEE Trans. Plasma Sci. 26, 290 (1998); A. Valfells, L. K. Ang, Y. Y. Lau, and R. M. Gilgenbach, Phys. Plasmas 7, 750 (2000); R. B. Anderson, W. D. Getty, M. L. Brake, Y. Y. Lau, R. M. Gilgenbach, and A. Valfells, Rev. Sci. Instrum. 72, 3095 (2001).

${ }^{5}$ A. Neuber, D. Hemmert, H. Krompholz, L. Hatfield, and M. Kristiansen, J. Appl. Phys. 86, 1724 (1999).

${ }^{6}$ J. R. M. Vaughan, IEEE Trans. Electron Devices 35, 1172 (1988).

${ }^{7}$ R. A. Kishek, Ph. D dissertation, University of Michigan, Ann Arbor, MI, 1997; R. A. Kishek and Y. Y. Lau, Phys. Rev. Lett. 80, 193 (1998).

${ }^{8}$ I. Gorelov, J. Lohr, R. W. Callis, W. P. Cary, D. Ponce, R. I. Pinsker, H. Chiu, and F. W. Baity, Bull. Am. Phys. Soc. 46, No. 8, 302 (2001).

${ }^{9}$ K. Felch, M. Blank, P. Borchard, P. Calahan, S. Cauffman, T. S. Chu, and H. Jory, IEEE Int'l Vacuum Electronics Conference, Monterey, CA, 2002, p. 332 (IEEE Cat. No. 02EX524); G. Dammertz, ibid., p. 330; V. E. Myasnikov, ibid., p. 334; K. Sakamoto, ibid., p. 336.

${ }^{10}$ H. Bosman and Y. Y. Lau, Bull. Am. Phys. Soc. 46, No. 8, 214 (2001).

${ }^{11} \mathrm{~S}$. Ramo, J. R. Whinnery, and T. Van Duzer, Fields and Waves in Communication Electronics, 3rd ed. (Wiley, New York, 1994), p. 290.

${ }^{12}$ J. D. Krauss and D. A. Fleisch, Electromagnetics with Applications, 5th ed. (McGraw-Hill, New York, 1999), p. 197.

${ }^{13} \mathrm{~T}$. V. George (private communication).

${ }^{14} \mathrm{~A}$. Theiss, (private communication).

${ }^{15}$ R. Heidinger, G. Dammertz, A. Meier, and M. K. Thumm, IEEE Trans. Plasma Sci. 30, 800 (2002). 